\title{
The Analysis on the Public Training of Mapping Knowledge Domain Based on Citespace III
}

\author{
Han-Lei XU1, a , Wei LI', b, \\ ${ }^{1,2}$ School of Economics and Management, Harbin Engineering University, China \\ axuhanlei@hotmail.com, byixiqianlv@163.com
}

Keywords: Public training, Mapping knowledge domain, Visualization analysis, Citespace III,

\begin{abstract}
Public training is one of significant measures to improve the quality of people's livelihood and raise the level of public services. Selecting the related literature on public training downloading from Web of science as the data source, using visualization analysis function of Citespace III, literature information would be converted into precise mapping knowledge domain. Through further analysis of mappings, we would know the theoretical framework and forefront in the field of public training. In recent years, in the field of public training, the Harvard University, Columbia University and the School of hygiene and tropical medicine of London University rank among the world. Over the past 15 years, most scholars pay attention to public health prevention training especially in mental and public health prevention training would still be the hot point in the future.
\end{abstract}

\section{Introduction}

Training system establishment is the most critical part of human resource exploitation. In addition to professional talents needed by national economy departments, other training systems become increasingly important for the promotion of comprehensive quality of our country including popularization of public health facilities, job training, and popularization of public consciousness on disasters. Public training based on social welfare is dominated by governmental departments and non-profit organizations and aimed to improve the level of public services and the national comprehensive quality [1].

Different from corporate training, public training is non-profit and object-nonspecific. Since the training object is the social public, the contents and forms of public training are universal, intelligible and identifiable. Another characteristic of public training is the diversification of organizations, since public training is usually sponsored by several departments, rather than a single institution [2]. Currently, the main tasks of public training in China, including policy propaganda, popularization of scientific and technological knowledge, popularization of compulsory education, and propaganda of health and hygiene knowledge, are conducted in various forms by governmental departments and aimed to improve the comprehensive quality of civils. Public training is of high research significance in the long run, since it is a necessary public measure for improvement of living quality and public service level and for benefiting the nation and the people. As for our country which is under the public service transition, public training is very necessary and should be urgently studied.

Faced with abundant academic data, many researchers are unable to identify the cutting-edge and systematic data, or even are unclear about the research hotspots. With the information visualization analysis software, we can easily and systematically extract the most valuable information and research hotspots. The term 'information visualization', first used in 1989, is still at the developing stage, but has been modestly applied in the fields of information management and statistics [3]. The information visualization analysis is to abstract information and statistics by using the computer interactive analysis mode and thus to display the between-node correlations on mappings simply and clearly. These mappings are rich in valuable information and we call them mapping knowledge domains [4]. Mapping knowledge domain help researchers to rapidly acquire useful information, which avoids the searching of massive literatures. 


\section{Data source}

The core collections on Web of Science (WOS) from Science Citation Index (SCI) were used as data sources. Data were precisely searched through advanced search of the term of "public and training'. The highly-cited literatures were selected. WOS (including SCI and SSCI, A\&HCI) is a globally-leading literature index database. Relying on unique citation retrieval mechanism and powerful intersective searching functions, WOS effectively integrates academic journals, technology patents, proceedings, academic analysis, evaluation tools, and other important academic information resources. WOS is a consistently-approved powerful database, and covers natural science, social science, arts and liberal arts. Data extraction from WOS guarantees the reliability and authenticity of the data source.

\section{Tools}

The information visualization technology helps to easily observe, browse and understand the information and to find the rules and patterns hidden in the data. Citespace, a Java-based application developed by the Chaomei Chen professor who is Changjiang Scholar from Dalian University of Technology, can display the abstracted data in the visual form and facilitates further data analysis, rule discovery and decision-making. Citespace is a new-generation information visualization software for multivariable, time-sharing and dynamic analysis of complex networks. This software becomes a new widely-used means in metrology. Citespace is unique because it uses the visualized analysis of scientific literatures to monitor hotspots, trends, directions and other patterns that appear in literatures [5]. In this paper, based on SCI and Citespace, we determined the discipline layout and hotspots about public training in a specific period of time. We used the latest version of Citespace III, to analyze related literatures.

\section{Data Collection}

The data source was the SCI literatures from the 2001-2015 WOS core collection. According to the protocol of Citespace III, we restricted the search title as 'public training'. Then from the initially-identified articles, we screened out the 231 highly-cited literatures. The highly-cited literatures further improved the value of data references.

To facilitate the operation of software algorithms, we obtained the plain-text data containing full records and references from WOS, and saved the data in the format of download_*.txt. With the data conversion function from Citespace, we partly saved the unconverted data and the converted data in the two files of input and output, which helped to conveniently identify the data paths. Citespace III provides conversion functions for many commonly-used databases, such as CSSCI and CNKI.

\section{Results}

After data conversion, we could set the parameters. Citespace III, showed out precise and satisfactory results, thanks to the precise prior conditions. The year span amplitude of data can be restricted depending on time. From the 11 types of analytical objects, we could select the objects for our need, such as author-cooperation and institution-cooperation. According to the specific situation, we could tick different algorithms in the dialog box and use them for moderate trimming, which avoids the occurrence of chaotic mappings. After parameter setting, we clicked the button'go' and got the mapping knowledge domains. Fig 1 shows the interface of Citespace III,. 


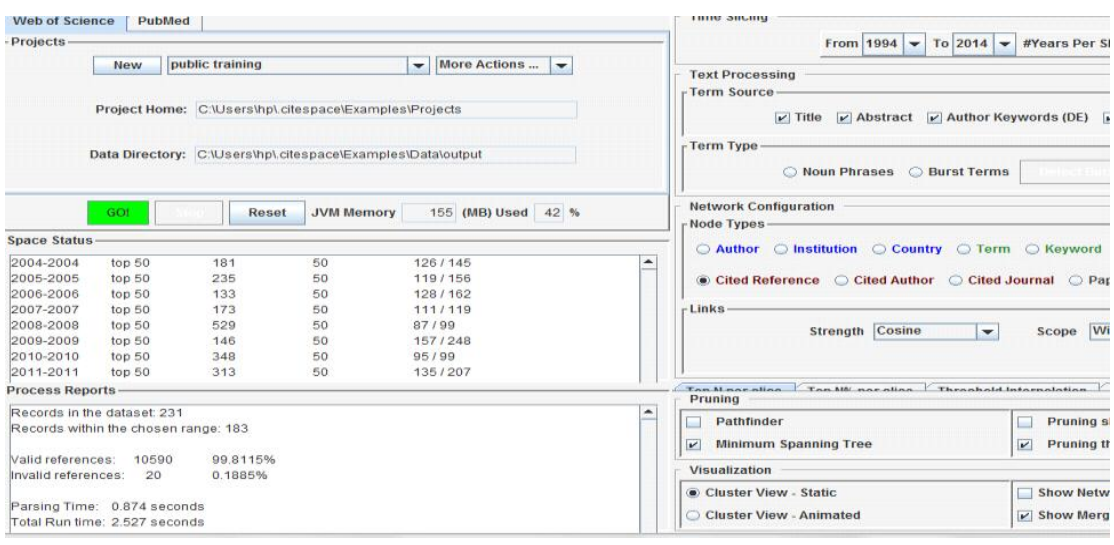

Fig 1 the interface of Citespace III,

WOS allows to create citation reports from the search results. From the citation reports, we can clearly identify the changes in the number of published literatures and the number of citations in recent years, and thereby roughly understand the developing trends in a specific year (Fig 2). Clearly, the annual number of published literatures about public training is generally rising in recent 15 years, and is maximized in recent five years. Moreover, the number of citations gradually rises in recent 15 years and peaks in 2005. Mappings analysis shows that public training has attracted growing attention from researchers, and the number of achievements is on the rise.
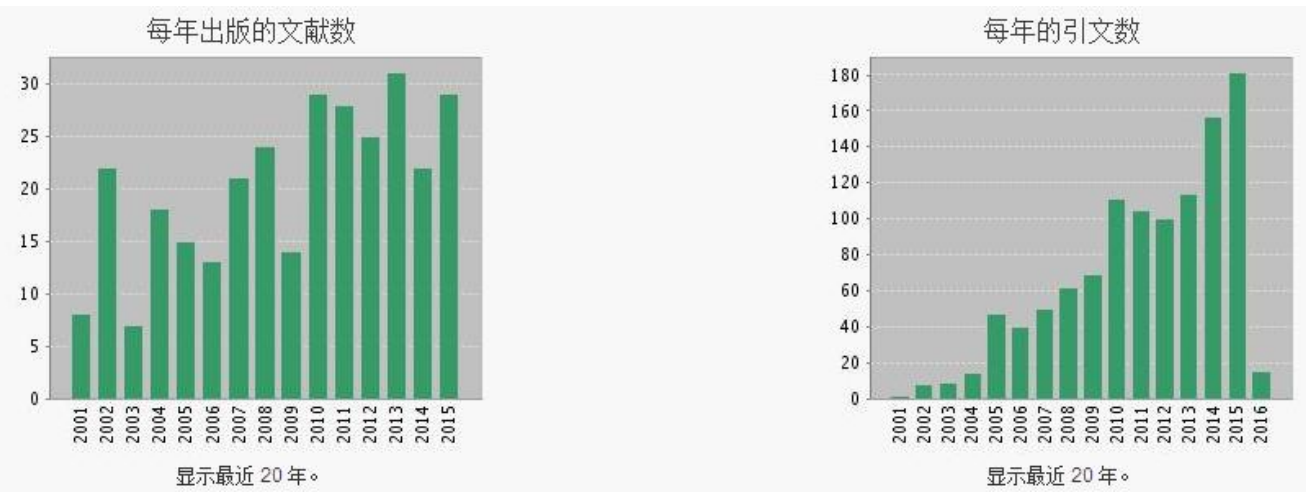

Fig 2 citation reports

\section{Mapping Knowledge Domains of Country-Level Cooperation}

With 'country' as the object of comprehensive analysis, we obtained the mapping knowledge domains of country-level cooperation. Then we modestly trimmed the mappings and regulated the labels. At last, we got the Fig 3.

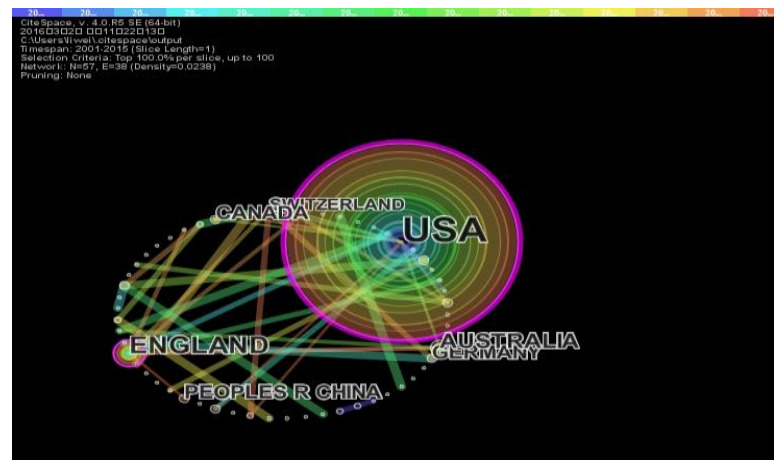

Fig 3 the mapping knowledge domains of country-level cooperation 
The tree-ring structure of nodes represents the history of a literature cited by scholars. The blue tree-rings denote the early years, and the red tree-rings denote the late years, while the tree-ring thickness is proportional to the cited frequency of this year. Thus, the radius of a node corresponds to the total number of citations of this node. From the mappings, we see the two most obvious nodes are 'US' and 'UK'. The tree-rings of node 'US' not only span over long time, but also are very thick, indicating the high cited frequency. The node centricity is up to 0.21 (far larger than 0.1 ), suggesting the US is at a critically important place in the field of public training. The tree-rings of node "UK" are not very thick, but the between-node connection is obviously strong, and the centricity is up to 0.12 , suggesting the second intermediary role of the UK only after the US. Moreover, the US and the UK, as the research centers of public training, account for the majority of cooperative academic projects. In both natural science and social science, the US and UK are always at the dominant positions in academy. If someone wants to probe into public training, s/he is recommended with the achievements coming from the US and the UK.

\section{Mapping Knowledge Domains of Institutional Cooperation}

Though we identified the UK and the US as the keys from the above analysis, it is not enough. Then we further explore the mapping knowledge domains of institutional cooperation (Fig 4). With 'Institution' as the object of comprehensive analysis, we obtained the mapping knowledge domains of institutional cooperation. Since the number of academic institutions is far larger than that of countries, after label adjustment, we can focus on the first few academic institutions with large label size and high obviousness.

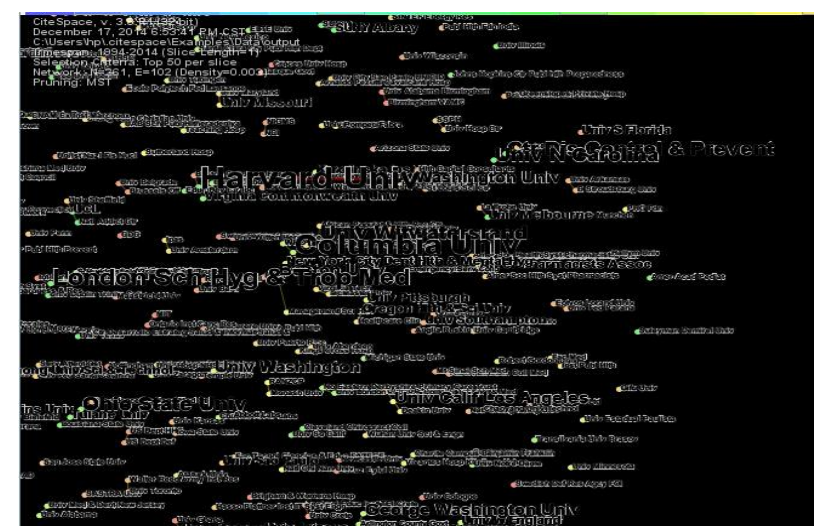

Fig 4 the mapping knowledge domains of institutional cooperation

From the mapping knowledge domains of institutional cooperation, we find the betweeninstitution cooperation relationship is not very obvious and the node labels are very scattered. The node with the most obvious label is Harvard University. Harvard has the largest comprehensive strength in the world, and plays a super dominant role in public training, and has made tremendous contributions. The second label, which is relatively smaller, is Columbia University in the City of New York. Columbia University not only ranks top in the world, but its social science also ranks top ten in the world, so it has a loud voice in the field of public training. London School of Hygiene \& Tropical Medicine, University of London, is also an evident node. Though the overall strength of University of London is inferior to Harvard or Columbia University, the London School of Hygiene \& Tropical Medicine has written many excellent literatures on health and medicine and thus attracts high attention from the world. Therefore, the research in public medical and health from this School is of considerable reference values for training.

\section{Co-occurrence Analysis of Keywords}

The research hotspots of a specific period are certainly reflected in academic journals, while the 
keywords of highly-refined literatures represent the research trends of an article. The occurring frequency and extent research of keywords highly reveal the state-of-the-art of a specific field. Cooccurrence analysis of keywords conducted on CitespaceIII are showed in Fig 5-1, Fig 5-2. We observe the state-of-the-art hotspots from the mappings of auto-clustering.

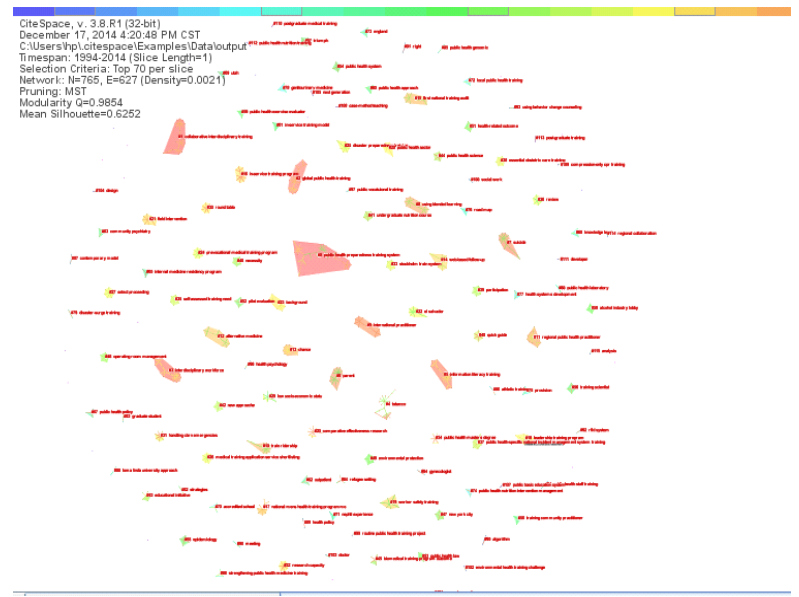

Fig 5-1 co-occurrence analysis of keywords

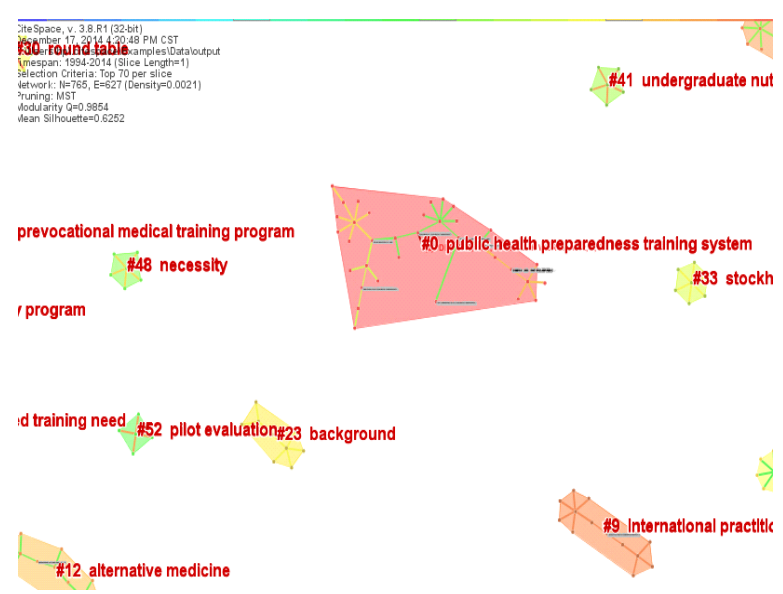

Fig 5-2 co-occurrence analysis of keywords

The literatures of the same cluster are largely similar in terms of themes. Depending on the shapes, sizes and distributions of clusters, we can easily differentiate the research key points and the discipline layout in a specific field. Clearly, the keywords in public training are very scattered (Fig 5-1), while the local magnification (Fig 5-2) shows the significant clusters of keyword '0\#public health preparedness training system'. The cluster 0\# is combined from the citing articles in many fields associated with public health. Its nodes are either dark or bright, suggesting this hotspot spanned very long time. In this cluster, the most highly-cited article is 'Mental health first aid training for the public: evaluation of effects on knowledge, attitudes and helping behavior' [6], written by B A Kitchener and A F Jorm and published on a world-famous journal BMC Psychiatry. A number of citizens with low psychological quality and mental fragility are unable to sustain diverse impacts from the society. Mental health first-aid training course was developed to improve public mental health. This article elaborates the contents of this course, and assesses the effects of knowledge, attitudes and helping behavior on training, and concludes that this training course largely helps to improve public mental health. This article provides some basic knowledge for research on public mental health and is the foundation in this field. B A Kitchener has made outstanding achievements in the field of psychiatry, and is the earliest ancestor in public mental health training. He also has many other highly-cited literatures, which indicate researchers have paid high attention to public mental health. 'Cardiopulmonary resuscitation training in Washington state public high schools' by S Reder and L Quan in Resuscitation is also a foundation literature [7]. It is stated that popularization of cardiopulmonary resuscitation (CPR) training in many state public high schools was inhibited due to the lack of fund and professional instructors, which became a threat to public health. This flaw should be faced up to and handled by governments and related institutions. Based on the achievements of S Reder, Nishiyama Chika et al. published 'Effectiveness of simplified chest compression-only CPR training for the general public: A randomized controlled trial' also on Resuscitation [8]. They experimentally compared the conventional CPR and a simplified CPR. They conclude the simplified CPR could be more easily popularized in public training, but has an equivalent effect as the traditional CPR. This article facilitates the popularization of public medical knowledge. J B Nachega et al. published 'Current status and future prospects of epidemiology and public health training and research in the WHO African region' in International Journal of Epidemiology, which inherits fine traditions and ushers in the future mission[9]. In this iconic literature, they analyzed and prospected the epidemic diseases and public health training in Africa, and underlay the research on public health training in recent years. These 
iconic literatures play a strong intermediary role in the largest cluster -- public health prevention training systems, underlie other clusters and are major references for deeper research. Besides the largest cluster-- public health, many small clusters are also related to public health, such as '\#12alternative medicine', and '\#48 prevocational medical training program necessity'. The cooccurrence analysis of keywords shows that public health preventive training system is a hotspot in the field of public training, while public mental health is the focus among all hotspots.

\section{Summary}

By analyzing the mapping knowledge domains of diverse disciplines related to public training on Citespace III, we clearly identify the overall discipline layout and research hotspots.

(1) Academic achievements about public training have spawned in recent 15 years. The US and UK are dominant in this field. Harvard University and Columbia University as the world's academic centers have contributed outstanding literatures. London School of Hygiene \& Tropical Medicine is also critical and has completed excellent achievements in public medical and health affairs.

(2) The co-occurrence analysis of keywords shows that public health prevention training becomes a focus of public training. In particular, the most highly-cited article is related to public mental health training, suggesting public mental health is attracting high attention from researchers and is the focus of all hotspots. As for public health, the highly-cited articles include 'Mental health first aid training for the public: evaluation of effects on knowledge, attitudes and helping behavior' by $B$ A Kitchener, 'Cardiopulmonary resuscitation training in Washington state public high schools' by $\mathrm{S}$ Reder, 'Effectiveness of simplified chest compression-only CPR training for the general public: A randomized controlled trial' by Chika Nishiyama, and 'Current status and future prospects of epidemiology and public health training and research in the WHO African region' by $J B$ Nachega.These four articles and the authors' other articles are basic literatures in public health prevention training and deserve thorough study. With growing attention to public health, this theme would attract more attention and is expected to be a hotspot in public training in the future.

\section{Acknowledgement}

This research was financially supported by the Fundamental Research Funds for the Central Universities under [Grant HEUCF150905]; and National Social Science Fund under [Grant 14AGL004].

\section{References}

[1] Xican He. Public training: basic project for development of rural human resources [J]. Social Construction, 2009, 3: 47-51.

[2] Hui Zhang, Zhansai Zhang, Xiaoyan He et al. Response capability of training and drilling to public health emergencies [J]. Chinese Journal of Health Inspection, 2011, 3: 249-252.

[3] B Bederson, B Shneiderman .The craft of information visual-ization: readings and reflections [M]. San Francisco: Morgan Kaufmann Publishers, 2003: 1-2.

[4] Yue Chen, Chaomei Chen, Liu Zeyuan et al. The methodology function of CiteSpace mapping knowledge domains) [J]. Studies in Science of Science, 2015: 33(2): 242-253.

[5] Chaomei Chen, Yue Chen .Zhigang Hu et al. Principles and Applications of Analyzing a Citation Space[M]. Beijing: Science Press, 2014: 14-15.

[6] B A Kitchener, A F Jorm Mental health first aid training for the public: evaluation of effects on knowledge, attitudes and helping behavior [J].BMC Psychiatry, 2002, 2.

[7] S Reder, L Quan. Cardiopulmonary resuscitation training in Washington state public high schools [J]. Resuscitation, 2003, 56(3):283-288. 
[8] Chika Nishiyama, IwamiTaku, Takashi Kawamura. Effectiveness of simplified chest compression-only CPR training for the general public: A randomized controlled trial [J]. Resuscitation, 2008, 79(1):90-96.

[9] J B Nachega, O A Uthman, Yuh-Shan Ho. Current status and future prospects of epidemiology and public health training and research in the WHO African region. International Journal of Epidemiology.2012, 41(6):1829-1846. 